\title{
Specific Neurotrophic Factors Support the Survival of Cortical Projection Neurons at Distinct Stages of Development
}

\author{
Lisa A. Catapano, Matthew W. Arnold, Francisco A. Perez, and Jeffrey D. Macklis \\ Division of Neuroscience, Children's Hospital, and Department of Neurology and Program in Neuroscience, Harvard \\ Medical School, Boston, Massachusetts 02115
}

\begin{abstract}
Repair of specific neuronal circuitry in the neocortex may be possible via neural precursor transplantation or manipulation of endogenous precursors in situ. These approaches will almost certainly require a detailed understanding of the mechanisms that control survival and differentiation of specific neuronal lineages. Such analysis has been hampered by the overwhelming diversity of neuronal types intermixed in neocortex and the inability to isolate individual lineages. To elucidate stagespecific controls over the survival of individual lineages of cortical neurons, we purified immature callosal projection neurons (CPN) at distinct stages of development from embryonic and postnatal mouse cortex by retrograde fluorescence labeling, followed by fluorescence-activated cell sorting. Purified CPN survive well in culture, acquire stage-specific projection neuron morphologies, and express appropriate neurotransmit-
\end{abstract}

ters and growth factor receptors. Purified CPN are dependent on exogenous trophic support for survival in a stage-specific manner. Survival of postnatal day 2 (P2) to P3 and P6-P7 CPN is promoted by overlapping but distinct sets of neurotrophic factors, whereas embryonic day 19 CPN show less specificity of dependence on peptide factors. These studies demonstrate for the first time the stage-specific control by peptide growth factors over the survival of a specific cortical neuronal lineage. Such information may be critical for the future goal of directed differentiation of transplanted or endogenous precursors toward cellular repair of complex cortical circuitry.

Key words: cortex; neocortex; callosal projection neuron; fluorescence-activated cell sorting; FACS; survival; neuronal culture; growth factors; neurotrophins
Reconstruction of complex neocortical circuitry may be possible via transplantation or manipulation in situ of defined populations of neural precursors. Neuronal replacement therapies will require the survival and directed differentiation of immature neuroblasts along desired lineages. Understanding the molecular factors that control the survival and differentiation of specific neuronal populations is therefore an important step in developing these therapies. Isolation of distinct neuronal populations from cortex may be necessary for the identification of such factors. However, lineage-specific neuron purification in the neocortex has been difficult, given the diversity and complexity of the neuronal populations in cortex.

Callosal projection neurons $(\mathrm{CPN})$ are prototypical cortical projection neurons that are especially vulnerable to dysgenesis or degeneration in a variety of conditions. Callosal projection neurons are excitatory pyramidal neurons in layers II/III and V of neocortex that project via the corpus callosum to targets in contralateral cortex. CPN and other long-distance cortical pro-

Received May 22, 2001; revised Aug. 21, 2001; accepted Sept. 4, 2001.

This work was supported by National Institutes of Health Grants HD28478 and NS41590, the Alzheimer's Association, the National Alliance for Autism Research, Mental Retardation Research Center (MRRC) Grant HD18655, and a Howard Hughes Medical Institute predoctoral fellowship to L.A.C. We thank Cindy Tai, Max Christian, and Witold Lipski for excellent technical support; Tene Cage for assistance with immunocytochemistry; and Dr. Paola Arlotta for advice and guidance with RT-PCR. We also thank Alan F. Flint of the MRRC Cell Sorter core facility for FACS technical support; Drs. Rosalind A. Segal, Louis F. Reichardt, John Rudge, and David R. Kaplan for generously providing antibodies; Drs. Gabriel Corfas, Rosalind A. Segal, and Charles D. Stiles for helpful discussions; and Drs. Larry I. Benowitz, Zhigang He, Qiufu Ma, and Sanjay S. Magavi for critical reading of this manuscript.

Correspondence should be addressed to Jeffrey D. Macklis, 354 Enders Building, 320 Longwood Avenue, Boston, MA 02115. E-mail: jeffrey.macklis@tch.harvard.edu. Copyright (C) 2001 Society for Neuroscience $0270-6474 / 01 / 218863-10 \$ 15.00 / 0$ jection neurons share unique properties of morphology, biochemistry, and vulnerability in a number of neurodegenerative diseases, e.g., interhemispheric callosal neurons in Alzheimer's disease (Pearson et al., 1985; Hampel et al., 1998), corticobasal ganglionic neurons in corticobasal degeneration (Yamauchi et al., 1988), corticostriatal neurons in Huntington's disease (Sapp et al., 1999), and corticospinal neurons in amyotrophic lateral sclerosis (Jackson and Bryan, 1998). Cortical CPN appear to undergo abnormal development in autism spectrum disorders (Egaas et al., 1995; Piven et al., 1997). This selective vulnerability of cortical projection neurons may be attributable to their extraordinary metabolic demands, predominantly excitatory neurotransmitters, and unique trophic requirements at distant target sites or in their local microenvironment. The elucidation of stage-specific controls over CPN survival and differentiation may contribute to an understanding of the vulnerabilities of pyramidal neurons and enable neuronal replacement or support therapies for diseases affecting cortical projection neurons.

Neuronal replacement by transplantation or manipulation of endogenous precursors in situ is possible in the adult mammalian neocortex, under appropriate conditions. Previous work in our laboratory (Macklis, 1993; Sheen and Macklis, 1995; HernitGrant and Macklis, 1996; Snyder et al., 1997; Leavitt et al., 1999; Sheen et al., 1999; Shin et al., 2000) has shown that immature neurons and multipotent neural precursors, transplanted into regions of adult mouse cortex undergoing targeted apoptotic projection neuron degeneration, can selectively migrate to areas of neuronal loss, differentiate appropriately into projection neurons, express appropriate neurotransmitters, and re-form specific long-distance projections. Similarly, endogenous neural precursors can be induced in situ to undergo neurogenesis and differ- 
entiate into mature projection neurons in adult mouse cortex undergoing targeted projection neuron death (Magavi et al., 2000). In the avian forebrain, induction of targeted neuronal death of projection neurons in zebra finch song circuitry causes increased replacement of behaviorally functional projection neurons from endogenous neural precursors in a system already undergoing low-level neurogenesis (Scharff et al., 2000). However, both transplanted and endogenous neural precursors undergo directed differentiation with relatively low efficiency. Purification and transplantation of later-stage neuronal precursors or immature neurons leads to more efficient integration, repopulation, and reconstruction of lost circuitry (R. A. Fricker-Gates, J. S. Shin, L. A. Catapano, C. C. Tai, and J. D. Macklis, unpublished results). The identification of peptide factors that promote directed CPN survival and differentiation may lead to increased efficiency of cellular repair by precursors.

We developed an approach for the purification and culture of immature neocortical CPN to elucidate the controls over their survival and differentiation. We present here the purification of CPN from three specific stages of development in mouse: (1) embryonic day 19 (E19), when motor-sensory CPN axons first extend to the midline via the corpus callosum (Floeter and Jones, 1985); (2) postnatal day 2 (P2) to P3, when their axons begin to innervate contralateral cortex; and 3) P6-P7, when target innervation is complete and a subpopulation of cortical neurons undergo developmental cell death (Spreafico et al., 1995), presumably attributable in part to competition for limited concentrations of survival factors. Labeling and purification of CPN on the basis of their contralateral projections produces an essentially homogenous population of callosally projecting neurons. Purified CPN survive in coculture with cortical cells and acquire appropriate, cell type-specific morphology and expression of neurotransmitters and growth factor receptors. CPN in culture undergo apoptosis in the absence of exogenous trophic support, but this death is rescued by specific neurotrophic factors in a stage-specific manner.

\section{MATERIALS AND METHODS}

CPN labeling in vivo. CPN in one cortical hemisphere of E18, P1-P2, or P4-P5 C57BL/6 mice were labeled by injection of fluorescent microspheres (Lumafluor) into the projection fields in contralateral motorsensory cortex. The extremely limited diffusion of latex microspheres ensured that only neurons whose axon terminals were in the immediate area of the injection (within $\sim 150 \mu \mathrm{m}$ ) were labeled by the fluorescent microspheres (Macklis and Quattrochi, 1991). For embryonic injections, pregnant mice were deeply anesthetized with Avertin, and embryos were sterilely injected through the uterine wall. Each embryo received two to three injections of 32-64 nl into motor-sensory cortex. Postnatal mice were anesthetized by hypothermia and received 8-10 injections in motor-sensory cortex, with $40-60 \mathrm{nl}$ per injection site. Microspheres are taken up by axon terminals and retrogradely transported to the cell bodies, thus labeling contralaterally projecting CPN in layers II/III and $\mathrm{V}$ in the hemisphere opposite the injection site (see Fig. $1 A$ ). All animal studies were performed in accordance with institutional and federal guidelines.

CPN dissociation and purification. Twenty-four to $48 \mathrm{hr}$ after microsphere injection, the hemisphere contralateral to injection was removed, and motor-sensory cortex was dissected in cold dissociation medium (20 mM glucose, $0.8 \mathrm{~mm}$ kynurenic acid, $0.05 \mathrm{~mm}$ APV, $50 \mathrm{U} / \mathrm{ml}$ penicillin$0.05 \mathrm{mg} / \mathrm{ml}$ streptomycin, $0.09 \mathrm{M} \mathrm{Na}_{2} \mathrm{SO}_{4}, 0.03 \mathrm{M} \mathrm{K}_{2} \mathrm{SO}_{4}$, and $0.014 \mathrm{M}$ $\mathrm{MgCl}_{2}$ ). Retrogradely labeled cortex was enzymatically digested in dissociation medium containing $0.16 \mathrm{gm} / 1 \mathrm{~L}$-cysteine $\mathrm{HCl}$ and $11.7 \mathrm{U} / \mathrm{ml}$ papain at $37^{\circ} \mathrm{C}$ for $30 \mathrm{~min}$. Papain digestion was then blocked with dissociation medium containing $10 \mathrm{mg} / \mathrm{ml}$ ovomucoid (Sigma, St. Louis, MO) and $10 \mathrm{mg} / \mathrm{ml}$ bovine serum albumin (BSA) at room temperature. Neurons were mechanically dissociated to create a single cell suspension by gentle trituration in iced OptiMem (Life Technologies, Gaithersburg,
MD) containing $20 \mathrm{~mm}$ glucose and both $0.4 \mathrm{~mm}$ kynurenic acid and $0.025 \mathrm{~mm}$ APV to protect against glutamate-induced neurotoxicity. Microsphere-labeled CPN were purified from the cortical cell suspension by fluorescence-activated cell sorting (FACS) using a FACSVantage flow cytometer (Becton Dickinson, Mountain View, CA) (see Fig. 1B). Typically, a litter of embryonic or postnatal pups produced $\sim 1 \times 10^{5}$ viable purified CPN before manipulation and plating.

CPN culture. CPN were plated on poly-L-lysine-coated (Sigma) glass coverslips (Fisherbrand Scientific Microscope Cover Glass) at $\sim 4 \times 10^{2}$ cells per coverslip for E19 CPN and $1 \times 10^{3}$ to $2 \times 10^{3}$ cells per coverslip for postnatal CPN. These plating densities resulted in $\sim 2 \times 10^{2}$ live CPN per coverslip for CPN at all stages after $2 \mathrm{~d}$ in vitro (DIV) in conditioned medium (CM) (see below). CPN at this concentration did not have direct physical contact with one another for the first few days in vitro; after $2 \mathrm{DIV}$, a subpopulation was in close enough proximity to have direct contact. CPN were plated under the following conditions: (1) in serum-free medium (SFM) [(0.034 gm/1 BSA, $1 \mathrm{mM} \mathrm{L-glutamine,} 25 \mathrm{U} / \mathrm{ml}$ penicillin $-0.025 \mathrm{mg} / \mathrm{ml}$ streptomycin, $35 \mathrm{~mm}$ glucose, and $0.5 \%$ B27 (Life Technologies) in Neurobasal medium (Life Technologies)] as control; (2) in SFM with isolated cocultures of mixed cortical cells from early postnatal mice or rats (see below), separated by a $0.8 \mu \mathrm{m}$ membrane (Millipore, Bedford, MA); (3) in conditioned medium (SFM conditioned overnight by cortical cells); or (4) in SFM containing peptide growth factors at $25 \mathrm{ng} / \mathrm{ml}$ [NGF (Alomone Labs, Jerusalem, Israel), BDNF (Alomone Labs or Peprotech, Rocky Hill, NJ), neurotrophin-3 (NT-3) (Alomone Labs or Peprotech), NT-4/5 (Alomone Labs), glial cell linederived neurotrophic factor (GDNF) (Alomone Labs), CNTF (Alomone Labs), PDGF (Upstate Biotechnology, Lake Placid, NY), leukemia inhibitory factor (LIF) (Alomone Labs), IGF-1 (Peprotech), and basic FGF (bFGF) (Sigma) with heparin $(0.4 \mu \mathrm{g} / \mathrm{ml})]$, and/or forskolin $(5 \mu \mathrm{M}$; Sigma). In pilot experiments, CPN were plated directly on mixed cortical cells. For the initial survival experiments comparing the time course of CPN survival in SFM versus coculture (see Fig. $4 A$ ), SFM included N2 supplement (0.1\%; Life Technologies) in Basal Medium Eagle (BME) (Life Technologies) rather than B27 supplement in Neurobasal medium. In the experiments investigating whether excitotoxity contributes to CPN death in serum-free conditions (see Fig. $4 B$ ), glutamate receptor antagonists kynurenic acid $(1 \mathrm{mM})$ and/or APV $(0.05 \mathrm{mM})$ were added to the serum-free culture medium. In experiments investigating the physical size of the factors that mediate the survival activity of conditioned medium, size fractionation was performed using Centriplus centrifugal filter devices (Amicon, Beverly, MA). In experiments investigating the stability of the survival-promoting activity in conditioned medium, conditioned medium was incubated at $100^{\circ} \mathrm{C}$ for $5 \mathrm{~min}$.

Mixed neuronal and glial cells used for coculture or production of conditioned medium were prepared from P2-P8 mouse or rat neocortex, dissociated as above to create a single cell suspension $\left(2-3 \times 10^{6}\right.$ cells $/ \mathrm{ml}$ ) and plated on poly-L-lysine-coated $0.8 \mu \mathrm{m}$ membrane inserts (Millipore) in serum-containing medium [5\% fetal calf serum, $1 \mathrm{~mm}$ L-glutamine, $25 \mathrm{U} / \mathrm{ml}$ penicillin- $0.025 \mathrm{mg} / \mathrm{ml}$ streptomycin, $35 \mathrm{~mm}$ glucose, and $0.5 \%$ B27 (Life Technologies) in Neurobasal medium (Life Technologies)] for $6 \mathrm{~d}$. For glial-enriched cultures, mixed cortical cells were plated on uncoated plastic in SCM, medium was changed at 4 DIV, and cells were passaged at 7-8 DIV, resulting in $>95 \%$ glial cultures. SCM was replaced by SFM $24 \mathrm{hr}$ before use in coculture or conditioned medium experiments.

CPN survival assays. CPN survival was assessed by morphology, trypan blue exclusion, and exclusion of propidium iodide $(0.1 \mathrm{mg} / \mathrm{ml}$; Calbiochem, La Jolla, CA) at 2 DIV. For selected experiments, viable CPN were labeled with the vital dye calcein, and dead cells were visualized with ethidium homodimer EthD-1 (Live/Dead Viability/Cytotoxicity Kit; Molecular Probes, Eugene, OR). CPN were visualized by phasecontrast and/or differential interference contrast (DIC) microscopy. Each experiment involved comparison of CPN survival in one or more growth factors to survival in SFM and in CM. This resulted in more experimental repetitions in SFM $(n=9$ in E19; $n=16$ in P2-P3; and $n=$ 8 in P6-P7) and in CM $(n=9$ in E19; $n=16$ in P2-P3; and $n=8$ in $\mathrm{P} 6-\mathrm{P} 7)$ than in any of the individual growth factor conditions $(n=3-6$ in E19; $n=3-9$ in P2-P3; and $n=3-5$ in P6-P7). Experiments assessing P2-P3 survival in BDNF, NT-3, IGF-1, and forskolin were repeated additional times to investigate additivity. There was no correlation between number of experiments per condition and observed effect on CPN survival. Analysis of CPN survival was performed in a blinded manner. We used paired $t$ tests to compare the mean survival in a given culture 
condition at P2-P3 with the mean survival in the same condition at P6-P7.

Immunocytochemistry. CPN cultures were fixed immediately after plating or after 2 DIV and then characterized by immunocytochemistry using the following primary antibodies: microtubule-associated protein-2 (MAP-2) (1:100; Sigma); neuronal-specific nuclear protein (NeuN) (1: 100; Chemicon, Temecula, CA); neuron-specific enolase (NSE) (no dilution; Zymed, San Francisco, CA); neurofilament (NF) (1:500; Sternberger Monoclonals, Lutherville, MD); GFAP (Incstar, Stillwater, MN); glutamate (1:500; Incstar); aspartate (1:500; Sigma); GABA (1:500; Incstar); TrkB (1:250; Santa Cruz Biotechnology, Santa Cruz, CA); TrkA (1:2500; gift from L. F. Reichardt, University of California, San Francisco, CA); TrkC (1:200; gift from D. R. Kaplan, McGill University, Montreal, Canada); IGF-IR $\alpha$ (1:100; Santa Cruz); PDGFR-B (1:200; Upstate Biotechnology); CNTFR $\alpha$ (1:2000; gift of J. Rudge, Regeneron Pharmaceuticals, Tarrytown, NY); GDNF family receptor $\alpha-1$ (GFR $\alpha$-1) (Santa Cruz Biotechnology); Y490 (pan-phosphorylated Trk; 1:100; gift from R. A. Segal, Dana Farber Cancer Institute, Harvard Medical School, Boston, MA); and bromodeoxyuridine (BrdU) (1:1000; Accurate Chemicals, Westbury, NY). Unless otherwise noted, CPN were fixed with $4 \%$ paraformaldehyde (PFA) in PBS, washed, blocked in $5 \%$ BSA and $3 \%$ goat serum (with or without $0.3 \%$ Triton X-100 or $0.1 \%$ Tween 20), incubated overnight in primary antibody, washed, incubated in secondary antibody [1:200; anti-rabbit Alexa 546, anti-rat Alexa546, or anti-goat Alexa488] for 1-2 hr, washed, and mounted in Fluoromount (BDH Laboratory Supplies, Poole, UK). For neurotransmitter immunocytochemistry, CPN were fixed with $4 \%$ PFA- $0.5 \%$ glutaraldehyde at $37^{\circ} \mathrm{C}$ and blocked with $10 \%$ goat serum and $0.01 \%$ Triton X-100. For anti-BrdU immunocytochemistry, CPN were incubated in $2 \mathrm{M} \mathrm{HCl}$ before the initial blocking step. For the anti-pTrk (Y490) antibody, CPN were fixed in 4\% PFA in TBS with $2 \mathrm{~mm}$ vanadate (TBS-V), washed in TBS-V, and blocked in 5\% goat serum in TBS-V. For biotinylated secondary antibodies, the above protocol was followed, but CPN were incubated in $3 \% \mathrm{H}_{2} \mathrm{O}_{2}$ before the initial blocking step, and, after incubation in biotinylated secondary antibody $(1: 200)$, CPN were processed according to the peroxidase ABC kit (Vectastain; Vector Laboratories, Burlingame, CA) and visualized with DAB substrate (Pierce, Rockford, IL). For in vivo immunocy tochemistry, CPN were labeled by intracortical injection as described above, using the retrograde marker Fluoro-Gold (Fluorochrome Inc., Englewood, NJ). Twenty-four to $48 \mathrm{hr}$ later, pups were anesthetized by hypothermia, and whole brains were removed and post-fixed for $1 \mathrm{~d}$ in $4 \%$ PFA before immunocytochemistry. For experiments investigating receptor expression immediately after purification, CPN were plated on glass coverslips and allowed to adhere for $15 \mathrm{~min}$ before fixation. Appropriate positive and negative controls, including omission of the primary antibody, were used in all experiments to ensure specificity of staining.

Reverse transcription-PCR. RNA was prepared from FACS-purified CPN at E19, P2-P3, and P6-P7 immediately after sorting or from freshly dissected P2-P3 whole brain, using a Stratagene (La Jolla, CA) Microprep kit. cDNA was transcribed using random primers and Superscript II reverse transcriptase (Life Technologies) according to the instructions of the manufacturer. Reverse transcription (RT)-PCR was performed with Taq polymerase (Sigma) according to the instructions of the manufacturer, using the following primers to generate 500-600 nucleotide fragments for each gene: trkB (forward primer, 5'-CTGAAAAACAGCAACCTGCGGC-3'; reverse primer, 5' -CCTCTCACAGTGAATGGAATGCACC $-3^{\prime} ; T_{\mathrm{m}}=62^{\circ}$ ); trkC (forwardprimer, $5^{\prime}$-CTCTTCCGCATGAACATCAGTCAG-3'; reverse primer, 5'-GGGCATTCTTAGCAATGAGGGTG-3'; $T_{\mathrm{m}}=62^{\circ}$ ); IGF-IR (forward primer, 5'-CGATTCGGTGACTTCTGCTCAAATG-3'; reverse primer, 5'-GTGCCACGTTATGATGATTCGGTTC-3'; $T_{\mathrm{m}}=62^{\circ}$ ); GDNF receptor (forward primer, 5'-AAACCAACTTCAGCCTGACATCCG-3'; reverse primer, 5'-AAGAGCATCCCGTAGCTGTGCTTG-3'; $T_{\mathrm{m}}=61^{\circ}$ ); PDGFR (forward primer, 5'-TCTGTGATCGAGAATGGCTACGTGC-3'; reverse primer, 5'-TGGGTGACAGTTTTCGTGGACACC-3'; $T_{\mathrm{m}}=61^{\circ}$ ); FGFR1 (forward primer, 5'-CGAATTGGAGGCTACAAGGTTCGC-3'; reverse primer, 5'-TATACTCCCCCGCATCCTCAAAGG-3'; $T_{\mathrm{m}}=62^{\circ}$ ); CNTFR $\alpha$ (forward primer, 5'-TGAAGCCTGATCCTCCAGAAAACG3'; reverse primer, 5'-TGACTGGGACACTGGTCAAGAAGAG-3'; $T_{\mathrm{m}}=$ $62^{\circ}$ ); and GFAP (forward primer, 5'-CCATGCCACGTTTCTCCTTGTCTC-3'; reverse primer, 5'-ATACGCAGCCAGGTTGTTCTCTGC$\left.3^{\prime} ; T_{\mathrm{m}}=62^{\circ}\right)$. Products were separated on a $1 \%$ agarose gel, and band intensity was quantitated using Kodak Digital Sciences software (Eastman Kodak, Rochester, NY). Products were compared quantitatively in the linear phase of the same PCR reaction, using purified CPN RNA or P2-P3 whole brain RNA as starting material. Glyceraldehyde-3phosphate dehydrogenase (GAPDH) was used as in an internal control for RNA intensity and quality.

\section{RESULTS}

\section{Callosal projection neurons can be purified by retrograde labeling and FACS}

CPN from E18, P1-P2, and P4-P5 mice were retrogradely labeled with green fluorescent latex microspheres injected into contralateral projection fields in neocortex (Fig. 1 $A$ ) and purified 24-48 hr later by dissociation of neocortex and FACS (Fig. 1B). This yielded a $>99.5 \%$ pure population of sorted neurons. Before FACS sorting, dissociated cortical cell populations typically contained 1-3\% fluorescently labeled callosal neurons; after purification, $\sim 90 \%$ of the purified cells were visibly fluorescent by microscopy (a much less sensitive method of detecting fluorescent labeling than FACS), and more sensitive cooled CCD digital imaging could detect fluorescence in most of the remaining cells (Fig. $1 C-F$ ). These resultant cultures of purified CPN were significantly enriched for markers of neuronal maturity and polarity. In unsorted dissociated cortical cell populations, $\sim 40 \%$ of cells were immunoreactive for MAP-2 and for NeuN, whereas in purified CPN cultures, $83 \%$ were immunopositive for MAP-2 (Fig. 2D) and $86 \%$ were immunopositive for NeuN. These purified CPN were also immunoreactive for NF (80\%) and NSE (77\%). Fewer than $0.5 \%$ of cells in the purified population expressed the glial marker GFAP by immunocytochemistry, in contrast to unsorted dissociated cortical cell populations, in which $\sim 40 \%$ of cells expressed GFAP. By RT-PCR, GFAP mRNA was barely detectable in $\mathrm{P} 2-\mathrm{P} 3$ purified cells compared with its high level of expression in mixed cells (neurons and glia) from P2-P3 whole brain (see Fig. 5I). Consistent with their identity as pure postmitotic neurons, purified $\mathrm{CPN}$ in vitro did not incorporate BrdU (0\% BrdU-positive P2-P3 CPN by immunocytochemistry).

Sorted CPN were reanalyzed by FACS. Of those CPN initially sorted, $88 \%$ were again above the fluorescence threshold for selection in a second round of FACS purification. These doublesorted CPN displayed similar survival responses to growth factors (see below) as did purified CPN from a single FACS purification.

\section{Purified CPN reextend axons and acquire stage-specific morphology and neurotransmitter expression in vitro}

To investigate whether CPN retained their in vivo pyramidal neuron phenotype after purification, we examined the morphology and expression of phenotypic markers of CPN in culture. Although CPN were axotomized as a result of dissociation and sorting (Fig. 2A), CPN reextended processes of up to $500 \mu \mathrm{m}$ (Fig. 2B,C) within 2 DIV in survival-promoting conditions. Consistent with the morphology of developing CPN in vivo, E19 CPN displayed multipolar, variable morphology (Fig. 2D,E), whereas postnatal CPN developed increasingly stereotypic unipolar pyramidal morphology (Fig. 2B, $C, F$ ).

Consistent with the neurotransmitter phenotype of neonatal CPN in vivo (Conti and Manzoni, 1994), the overwhelming majority of purified P2-P3 CPN were labeled by immunocytochemistry against glutamate (88\%), and a subpopulation expressed aspartate and/or GABA by immunocytochemistry (Fig. 3).

\section{Purified CPN survive in culture with appropriate trophic support}

To investigate the survival requirements of purified CPN, we assessed their ability to survive in culture in defined medium 

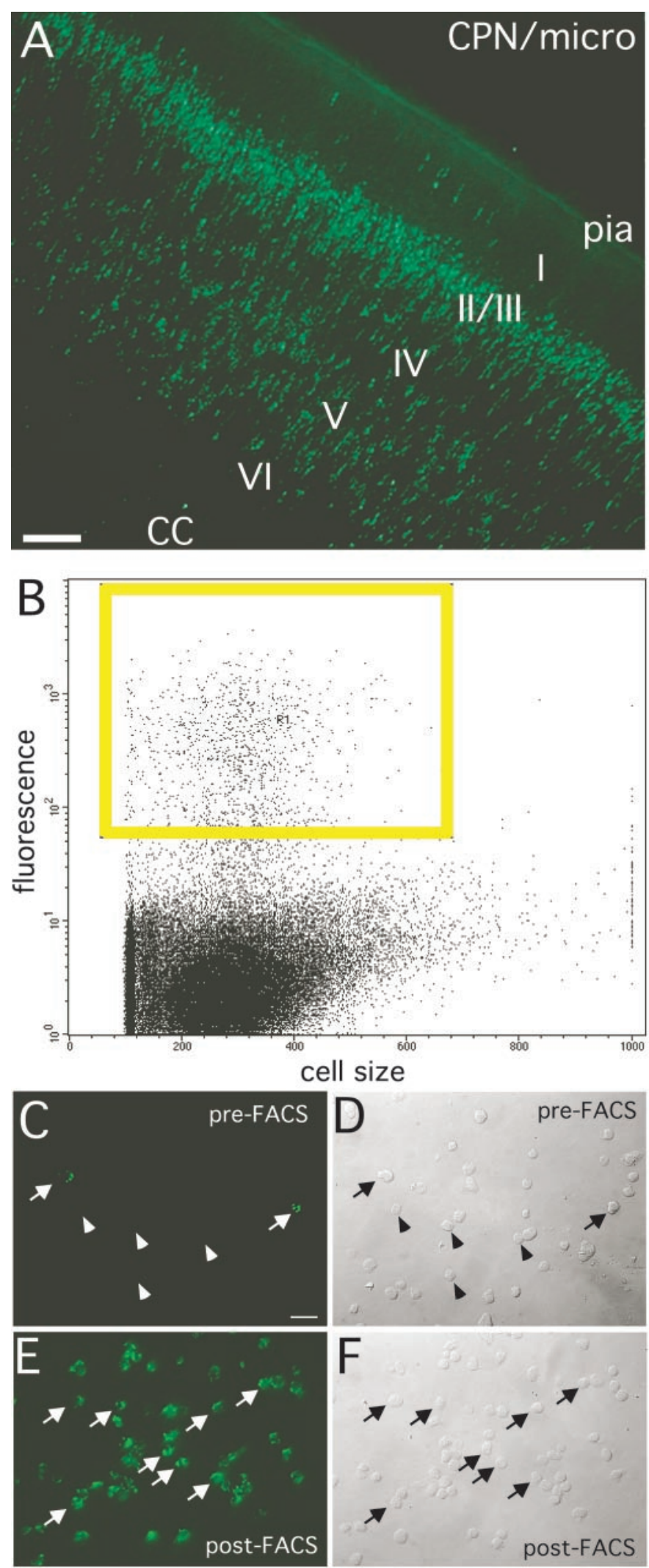

Figure 1. CPN purification by retrograde labeling and FACS. $A$, Lowmagnification fluorescence photomicrograph of $\mathrm{P} 2-\mathrm{P} 3$ neocortex, demonstrating green fluorescent microsphere-labeled CPN, primarily in layers II/III and V. Pial surface ( pia), cortical laminas (I-VI), and corpus callosum $(C C)$ are indicated. $B$, Sample FACS plot of the population of neurons selected. Cell size is represented on the $x$-axis, and fluorescence without exogenous trophic support. Purified P2-P3 CPN did not survive in BME-N2 SFM without the addition of trophic factors, in contrast to high levels of sustained neuronal survival in control cultures of mixed neurons and glia under the same conditions (Fig. 4A). This CPN death in serum-free conditions could be rescued by coculture with cortical neurons and glia (Fig. $4 A$ ). For the majority of our experiments, $C P N$ were maintained under these coculture conditions for 2-3 DIV; in pilot experiments, purified CPN survived well in these conditions for several weeks. In initial, pilot experiments, CPN were cultured in direct contact with mixed cortical cells ("direct coculture"). To investigate whether soluble factors produced by mixed cortical cells were capable of supporting CPN survival, we cultured CPN (1) separated from mixed cortical cells by a $0.8 \mu \mathrm{m}$ membrane ("isolated coculture") or (2) in serum-free medium conditioned by mixed cortical cells ("conditioned medium"). CPN survived well in both of these conditions ( $89 \pm 16$ vs $100 \pm 25$, respectively, normalized to survival in conditioned medium), indicating that mixed neurons and glia promote CPN survival via diffusible factors. Survival of CPN in medium conditioned by cortical glia alone was not significantly different from that in medium conditioned by mixed cortical cells ( $95 \pm 1.1 \mathrm{vs} 100 \%$ ), indicating that glia were able to produce survival-promoting factor(s).

To further investigate the characteristics of the survival activity of mixed cell-conditioned medium, we established that this survival activity of CM was heat-labile $(0 \% \mathrm{CPN}$ survival in heattreated conditioned medium) and $>10,000 \mathrm{kDa}$ molecular weight by size fractionation, consistent with the activity of $\mathrm{CM}$ being attributable to a protein or set of proteins.

We directly investigated the hypothesis that CPN death in SFM was attributable to an absence of required trophic support rather than death by trauma or excitotoxicity. Ethidium homodimer EthD-1 (Fig. 4A, inset), propidium iodide, and Hoechst staining all demonstrated progressive nuclear condensation, suggesting that P2-P3 CPN death in SFM was apoptotic in the absence of coculture or conditioned medium. This apoptotic death over 24-48 hr is consistent with a dependence on neurotrophic factor support. In additional support of this hypothesis, CPN purified from Bax $-/-$ mice displayed increased survival in SFM compared with wild-type CPN, and condensed nuclei were not observed by ethidium, propidium iodide, or Hoechst staining (L. A. Catapano and J. D. Macklis, unpublished observations). This death in serum-free medium was not blocked by glutamate receptor antagonists kynurenic acid and/or APV (Fig. 4B), indicating that this death was not attributable to excitotoxicity.

\section{Purified CPN in vitro express specific growth factor receptors}

To ensure that the FACS purification process did not strip CPN of their growth factor receptors (rendering CPN unable to re-

intensity is represented on the $y$-axis. The highlighted box within the plot denotes the subpopulation of dissociated cortical neurons with highintensity fluorescence labeling $(\sim 3 \%)$, indicating that they are retrogradely labeled with fluorescent microspheres. This population is distinct from the predominant population of unlabeled cells. $C-F$, FACS purification results in cell populations highly enriched for retrogradely labeled CPN. $C, D$, A small percentage (arrows) of dissociated cortical cells $(D)$ are labeled with green fluorescent microspheres $(C)$ before purification. Arrowheads indicate nonlabeled cortical cells. $E, F$, Essentially all FACSpurified cells $(F)$ are callosal projection neurons labeled with green fluorescent microspheres ( $E$, arrows). Scale bars: $A, 100 \mu \mathrm{m}$; (in $C$ ) $C-F$, $25 \mu \mathrm{m}$. 

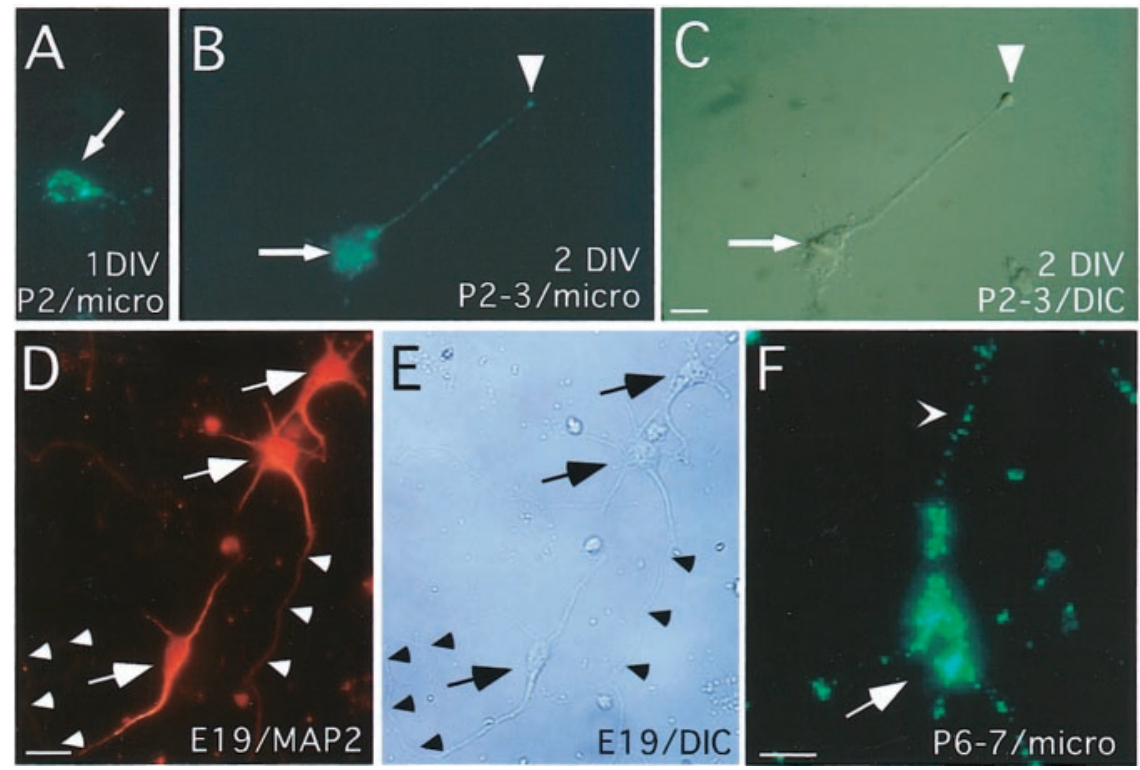

Figure 2. CPN morphology in vitro. A, FACSpurified CPN after 1 DIV, labeled with fluorescent microspheres and axotomized as a result of dissociation and cell sorting. $B, C$, FACS-purified CPN after 2 DIV, demonstrating early reextension of its axon. $A r$ rowhead indicates the growth cone. $B$, Fluorescence view of CPN displaying green microsphere labeling. $C$, The same neuron as in $B$ viewed by Nomarski DIC microscopy. $D, E$, FACS-purified E19 CPN, displaying variable, multipolar morphologies. $D$, E19 CPN acquire normal neuronal polarity, visualized with neuron-specific somatodendritic MAP-2 immunolabeling. $E$, The same neuron viewed by Nomarski DIC microscopy. Arrowheads indicate presumptive axons. $F$, FACS-purified P6-P7 CPN labeled with green microspheres, displaying stereotypical, mature unipolar pyramidal neuron morphology. Scale bars: (in $C$ ) $A-C$, $10 \mu \mathrm{m}$; (in $D$ ) $D, E, 20 \mu \mathrm{m} ; F, 10 \mu \mathrm{m}$.

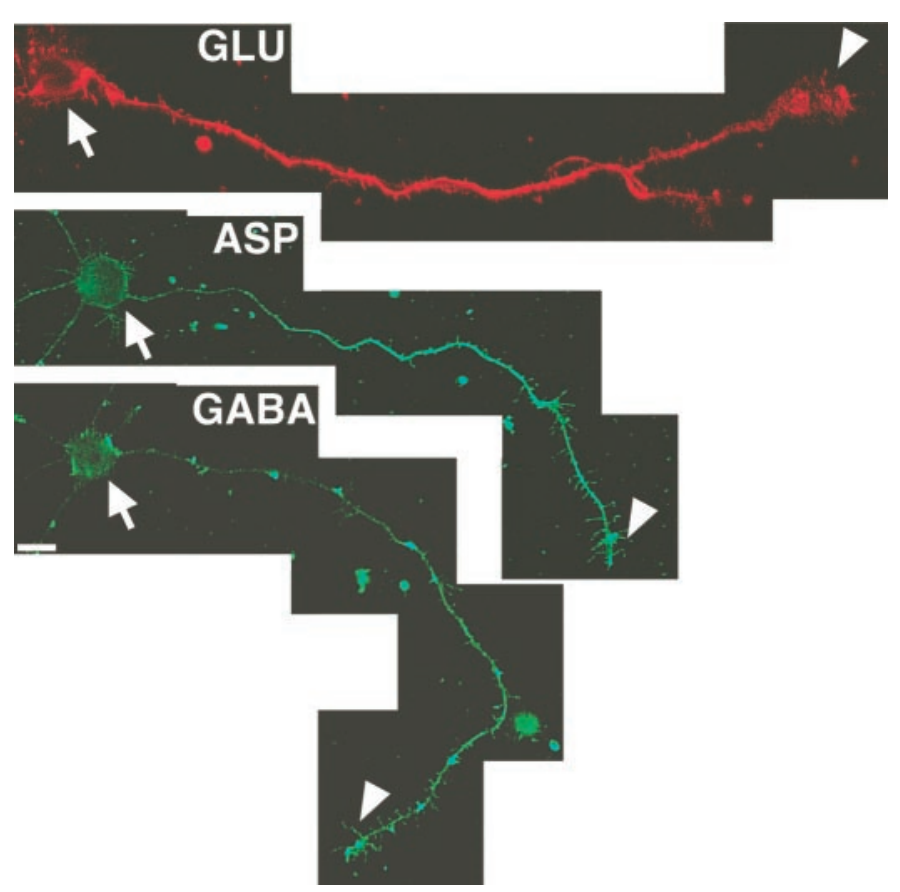

Figure 3. CPN neurotransmitter expression in vitro. Neurotransmitter expression by FACS-purified P2-P3 CPN, as assessed by immunocytochemistry. Large arrows indicate cell bodies. Arrowheads indicate growth cones at the axon terminals. Glu, Glutamate; Asp, aspartate. Scale bar, $10 \mu \mathrm{m}$.

spond to potential trophic effects of growth factors) or significantly alter the set of receptors normally expressed by CPN, we assessed receptor expression by immunocytochemistry immediately and $2 \mathrm{~d}$ after FACS purification. We also assessed CPN receptor expression in vivo and compared it with our in vitro results. $\mathrm{CPN}$ at $\mathrm{E} 19, \mathrm{P} 2-\mathrm{P} 3$, and $\mathrm{P} 6-\mathrm{P} 7$, in vitro and in vivo, expressed multiple growth factor receptors. At 2 DIV, postnatal CPN were labeled by immunocytochemistry against the neurotrophin receptors $\operatorname{TrkB}(39 \pm 17 \%)$ (Fig. $5 A)$, TrkA $(33 \pm 12 \%)$, and TrkC $(48 \pm 8 \%)$ and the growth factor receptors IGF-IR $\alpha$ $(35 \pm 16 \%)($ Fig. $5 D)$, GFR $\alpha-1(71 \pm 3 \%)$, and CNTFR $\alpha(41 \pm$ 9\%). CPN expressed PDGFR-B by immunocytochemistry at low levels. Individual $\mathrm{CPN}$ in vitro coexpressed multiple receptors, e.g., TrkB and IGF-IR $\alpha$. Consistent with their expression pattern in vitro, approximately half of $\mathrm{CPN}$ in vivo at $\mathrm{P} 2-\mathrm{P} 3$ and $\mathrm{P} 6-\mathrm{P} 7$ were labeled by immunocytochemistry against TrkB (Fig. 5B,C) and IGF-IR $\alpha$ (Fig. $5 E, F$ ), and a subpopulation coexpressed both receptors.

Receptor expression was confirmed immediately after purification. By immunocytochemistry, a subpopulation of CPN expressed surface receptors immediately after plating, including TrkB (Fig. 5G, $H$ ) and IGF-IR $\alpha$. By RT-PCR (Fig. 5I), CPN from E19, P2-P3, and P6-P7 strongly expressed trkB, trkC, IGF-IR, GFR $\alpha-1$, and CNTFR immediately after sorting, consistent with our immunocytochemistry data. PDGFR and FGFR1 were also detected at each age at low levels.

We confirmed that FACS purification did not adversely affect the function of receptors on the surface of CPN. Conditioned medium, or the neurotrophin BDNF alone, stimulated the phosphorylation of Trk receptors as assessed by immunocytochemistry with the pan-phosphorylated Trk antibody Y490 (data not shown), indicating that Trk receptors were able to be activated in culture after CPN dissociation and purification.

\section{Specific neurotrophic factors promote survival of purified neonatal CPN in vitro}

Although almost all purified neonatal $\mathrm{CPN}$ in vitro died in SFM, the addition of specific neurotrophic factors rescued this death (Fig. 6B). Compared with survival in CM, only $17 \%$ of P2-P3 CPN survived after 2 DIV in Neurobasal-B27 SFM. BDNF, NT-3, NT-4/5, IGF-1, and GDNF, added to SFM, promoted survival of purified P2-P3 CPN above that in SFM alone, whereas NGF, bFGF, PDGF, CNTF, and LIF had no significant survival effect. The effect of peptide factors was additive. For example, the combination of BDNF, NT-3, and IGF-1 promoted survival of purified P2-P3 CPN much better than any single factor and even significantly better than conditioned medium (Fig. 6B, inset). Forskolin, which elevates intracellular cAMP, also exerted a significant survival effect (Fig. 6B); its effect was additive with those of peptide factors (Fig. $6 B$, inset). Surviving CPN in CM or growth factors typically displayed healthy projection neuron morphology after 2-3 DIV (Figs. $2 D-F, 3,5 A, D, 6 A$ ). 


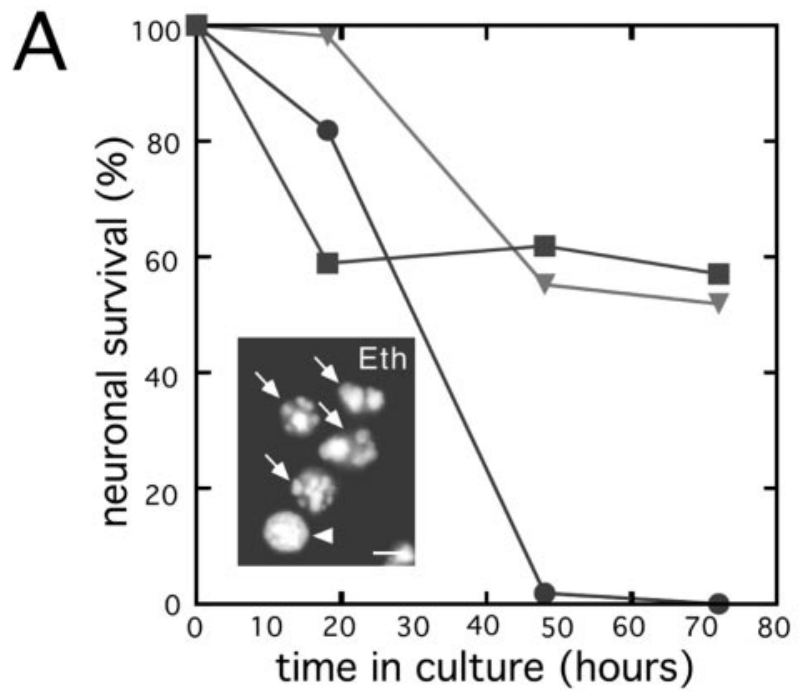

- control culture (mixed neurons and glia) - purified CPN alone in serum-free medium $\checkmark \mathrm{CPN}$ in co-culture with mixed cortical cells

$\mathrm{B}$

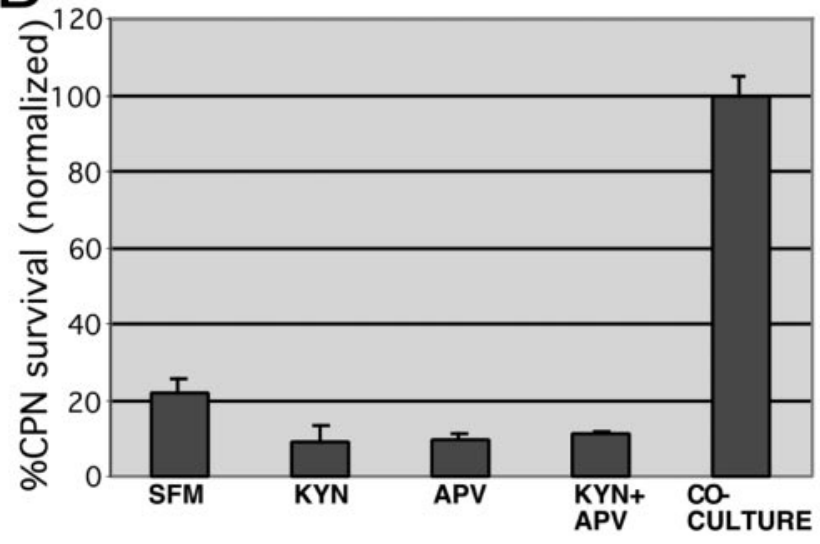

Figure 4. $\quad$ CPN survival in vitro. A, P2-P3 CPN die in serum-free medium without trophic support (circles), in contrast to control neurons in mixed cultures with glia (squares). CPN death can be rescued to control levels by coculture with mixed cortical cells (triangles). Survival values are normalized to immediate postplating survival (defined as 100\%). Inset, CPN undergo apoptosis with nuclear condensation (arrows), visualized with ethidium homodimer EthD-1 (Eth), in serum-free medium without trophic support. A neighboring nucleus without condensation (arrowhead) is shown for comparison. $B$, CPN death in SFM is not rescued by the glutamate receptor antagonists kynurenic acid $(K Y N)$ and APV. Scale bar: inset, $5 \mu \mathrm{m}$.

\section{Neurotrophic factors promote survival of purified CPN in vitro in a stage-specific manner}

The effects on survival exerted by neurotrophic factors were stage specific. Compared with P2-P3 CPN survival, P6-P7 CPN survival was promoted by an overlapping, but distinct, set of peptide factors. BDNF, NT-3, NT-4/5, IGF-1, GDNF, and PDGF significantly increased survival above that in SFM alone (26\% survival), but NGF, bFGF, CNTF, and LIF did not (Fig. 6C,D).

E19 CPN displayed much less dependence on exogenous growth factors for survival than did postnatal CPN. Survival of E19 CPN in CM was approximately fivefold higher than that of postnatal CPN, and E19 CPN survival in SFM was $71 \%$ of survival in CM (E19 survival vs P2-P3 survival, $p<0.001$; E19 survival vs P6-P7 survival, $p<0.001)$. E19 CPN survival in growth factors tested singly was $36-83 \%$ of survival in CM (Fig. $6 E$ ); no growth factors increased E19 CPN survival significantly above that in SFM alone. Combinations of growth factors did not promote E19 CPN survival better than did growth factors applied singly. E19 CPN survival in the combination of BDNF, NT-3, IGF-1, GDNF, and forskolin was only $68 \%$ of survival in CM, not significantly different from survival in the presence of each of these factors individually, or in SFM alone.

\section{DISCUSSION}

In these experiments, we investigated the stage-specific controls over the survival of neocortical CPN purified by FACS at three distinct stages during the development of this lineage: E19, when the first CPN axons extend to the midline; P2-P3, when the axons innervate contralateral target regions; and $\mathrm{P} 6-\mathrm{P} 7$, when target innervation is complete and a subpopulation of cortical neurons normally undergo developmental cell death. We demonstrated that, with appropriate methods and neuroprotection during the purification process, purified CPN survive and grow in pure culture for weeks. This allowed us to study the specific trophic requirements of these CNS neurons at each of these stages of development.

Our purification via retrograde labeling with latex microspheres, cortical dissociation, and FACS ensures that the only cells selected are those cortical neurons that project to contralateral cortex via the corpus callosum. This results in a nearly pure population of projection neurons: $<0.5 \%$ express GFAP, and the vast majority are immunoreactive for MAP-2, NeuN, NF, and NSE. Because these neuronal markers are known to recognize fewer than $100 \%$ of actual neurons by immunocytochemistry as a result of technical limitations of fixation, antibody titer, and unequivocal detection above background, these immunocytochemical results are consistent with our purification process yielding an essentially pure population of neurons. These purified callosal projection neurons are mostly homogenous, although variations in morphology and gene expression clearly exist within the population both in vivo and in vitro.

\section{Callosal projection neurons survive and retain their in vivo phenotype in culture}

After purification and plating, CPN retain their in vivo phenotype by multiple criteria. Many embryonic and neonatal $\mathrm{CPN}$ in vivo exhibit multiple projections, both contralateral and ipsilateral, but postnatally these neurons retract all but their single contralateral axonal projection (Ivy and Killackey, 1982). CPN in vitro display developing pyramidal neuron morphologies that mimic their stage-specific morphological phenotypes in vivo: multipolar and variable at E19 and stereotypically unipolar at P6-P7. CPN in culture also retain their neurotransmitter expression phenotype. In vivo, neonatal $\mathrm{CPN}$ express primarily glutamate, and, to a lesser extent, aspartate and GABA, although the latter is downregulated by the end of the first postnatal week (Conti and Manzoni, 1994). In vitro, P2-P3 CPN express this same set of neurotransmitters. Finally, we demonstrated that purified CPN in vitro retain their pattern of receptor expression and dependence on trophic factor support. CPN in vivo are dependent on targetderived neurotrophic factors, as evidenced by the increase in CPN death after callosotomy or the destruction of contralateral neocortex (Conti and Manzoni, 1994). Our data show that CPN in vitro express specific growth factor receptors and, in the absence 

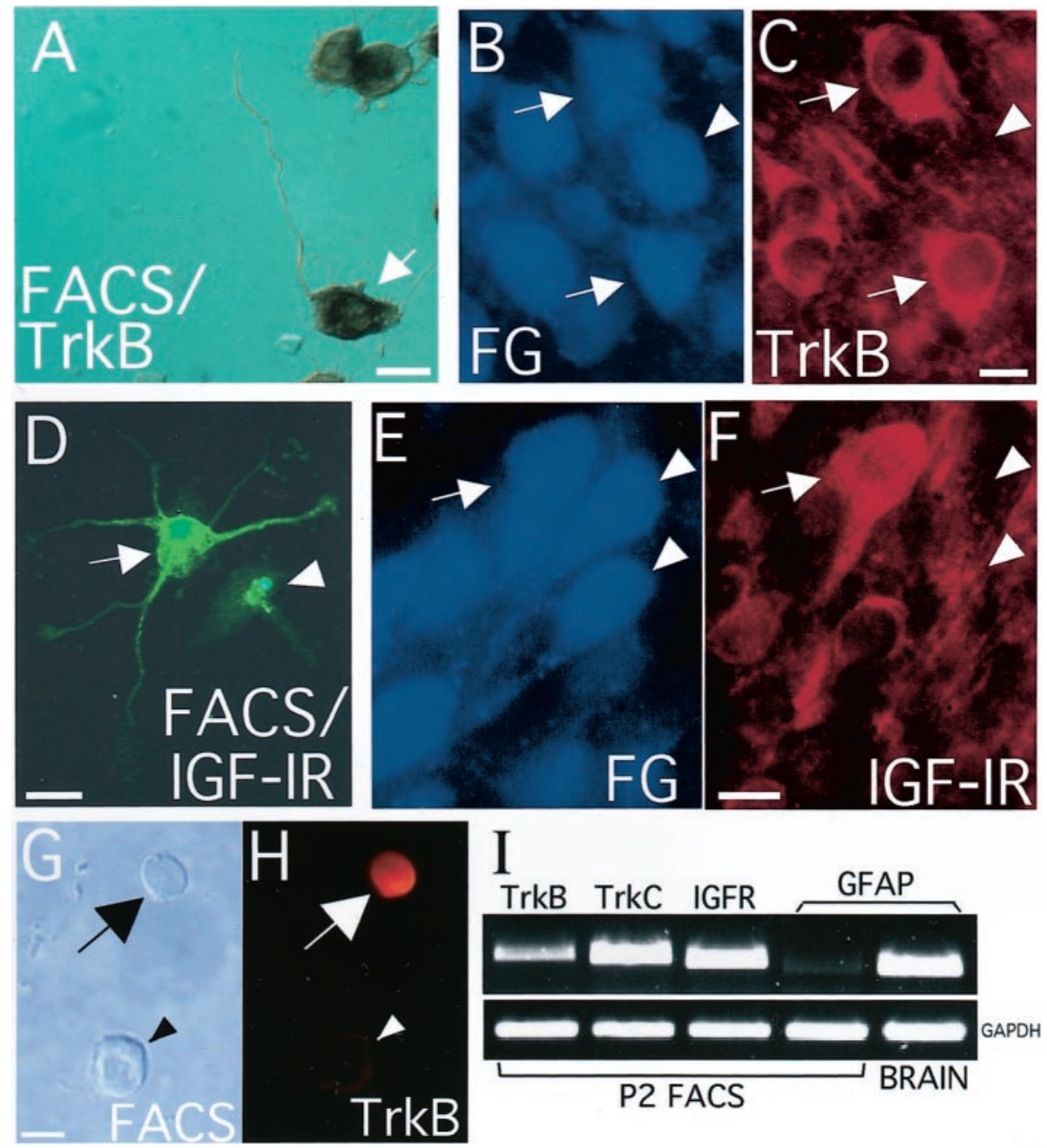

Figure 5. CPN growth factor receptor expression. A, Purified P2-P3 CPN express the neurotrophin receptor TrkB in vitro by immunocytochemistry. $B, C, \mathrm{P} 6-\mathrm{P} 7 \mathrm{CPN}$ express TrkB in vivo. $B, \mathrm{CPN}$ in somatosensory cortex, retrogradely labeled with Fluoro-Gold $(F G$; arrows and arrowhead). $C$, The same field as $B$, visualized by fluorescence microscopy after TrkB immunocytochemistry. Arrows indicate TrkBimmunolabeled CPN. Arrowhead indicates a CPN that does not label for TrkB. D, P2-P3 CPN in vitro, labeled by IGF-IR $\alpha$ immunocytochemistry (arrow). A second CPN is not labeled immunocytochemically, but low-level microsphere labeling is visible in this cell (arrowhead). E, F, P6-P7 CPN express IGF-IR $\alpha$ in vivo. $E, \mathrm{CPN}$ in somatosensory cortex, retrogradely labeled with Fluoro-Gold (arrow and arrowheads). F, The same field as $E$, visualized by fluorescence microscopy after IGF-IR $\alpha$ immunocytochemistry. Arrow indicates an IGF-IR $\alpha$-immunolabeled CPN. Arrowheads indicate CPN that do not label for IGF-IR $\alpha . G, H, \mathrm{P} 2-\mathrm{P} 3 \mathrm{CPN}$ express TrkB in vitro immediately after purification. $G, \mathrm{CPN}$ in vitro (arrow and arrowhead) viewed by Nomarski DIC microscopy. $H$, The same field as $G$, visualized by fluorescence microscopy after TrkB immunocytochemistry. Arrow indicates a TrkBimmunolabeled CPN. Arrowhead indicates CPN that does not label for TrkB. I, P2-P3 CPN express TrkB, TrkC, and IGF-IR receptors by RTPCR. RT-PCR was performed using cDNA from purified P2-P3 CPN (first four lanes) and from P2-P3 whole brain ( fifth lane), with primers specific to TrkB, TrkC, IGF-IR, and GFAP. GFAP mRNA was barely detectable in CPN compared with its level of expression in cells from P2-P3 whole brain, confirming the high degree of purification by FACS. All PCR products were 500600 bp. GAPDH was used as an internal control. Scale bars: $A, 10 \mu \mathrm{m}$; (in $C$ ) $B, C, 10 \mu \mathrm{m} ; D, 10$ $\mu \mathrm{m}$; (in $F$ ) $E, F, 10 \mu \mathrm{m}$; (in $G$ ) $G, H, 20 \mu \mathrm{m}$. of medium conditioned by neonatal cortical cells, undergo apoptosis. Specific neurotrophic factors alone and in combination promote CPN survival under these conditions, suggesting that these factors may be at least partially responsible for the control of CPN survival during development in vivo.

\section{Cortical glial-derived factors or neurotrophic factors promote survival of purified CPN}

Postnatal CPN display poor survival in serum-free conditions, indicating their dependence on factors derived from other cells and their inability to provide adequate trophic support for themselves. Coculture with, or conditioned medium from, mixed cortical cells is able to support CPN survival, indicating, as expected, that the cortical environment produces survival signals for these neurons. Apoptotic death in the absence of trophic support occurs over 24-48 hr and is reminiscent of that seen by PNS neurons after withdrawal of neurotrophic factors. Glutamate receptor antagonists did not block this death, indicating that it is not caused by excitotoxicity. In fact, this glutamate receptor blockade appears to modestly but reproducibly decrease survival (Fig. 4B), consistent with the hypothesis that activity plays a role in promoting CPN survival.

Our data show that several neurotrophic factors (BDNF, NT-3, NT-4/5, IGF-1, and GDNF) are able to partially support P2-P3 CPN survival, and P2-P3 CPN express the appropriate receptors for these ligands: TrkB, TrkC, IGF-IR, and GFR $\alpha-1$. This effect of these factors is specific, because other known neurotrophic factors (NGF, bFGF, PDGF, CNTF, and LIF) do not result in enhanced survival. The high degree of purification of these neurons allows us to conclude that these factors act directly on CPN. Furthermore, because CPN are axotomized during their isolation and FACS purification and gradually regrow neurites over several days, CPN have no direct connection with one another via neurites during most of the first $2 \mathrm{~d}$ in vitro. Therefore, the effects of the neurotrophic factors on survival are likely to be direct and not mediated by neuronal activity increased by the factors in a paracrine manner or by trophic factor production by other neurons. The additive effect of peptide factors on postnatal CPN survival, and the coexpression of multiple grow th factor receptors by CPN, suggest that CPN require multiple factors simultaneously for maximal survival.

In the peripheral nervous system, a number of classes of neurons, including sympathetic and dorsal root ganglion neurons, have been purified and characterized with respect to stagespecific neurotrophic requirements. However, because CNS neuronal subtypes have been difficult to purify, few have been characterized in this regard. Retinal ganglion cells (RGCs) have been purified on the basis of cell type-specific antigenicity and grown in culture (Barres et al., 1988); multiple neurotrophic factors have been identified that promote their survival in a stage-specific manner (Meyer-Franke et al., 1995). For embryonic and postnatal 

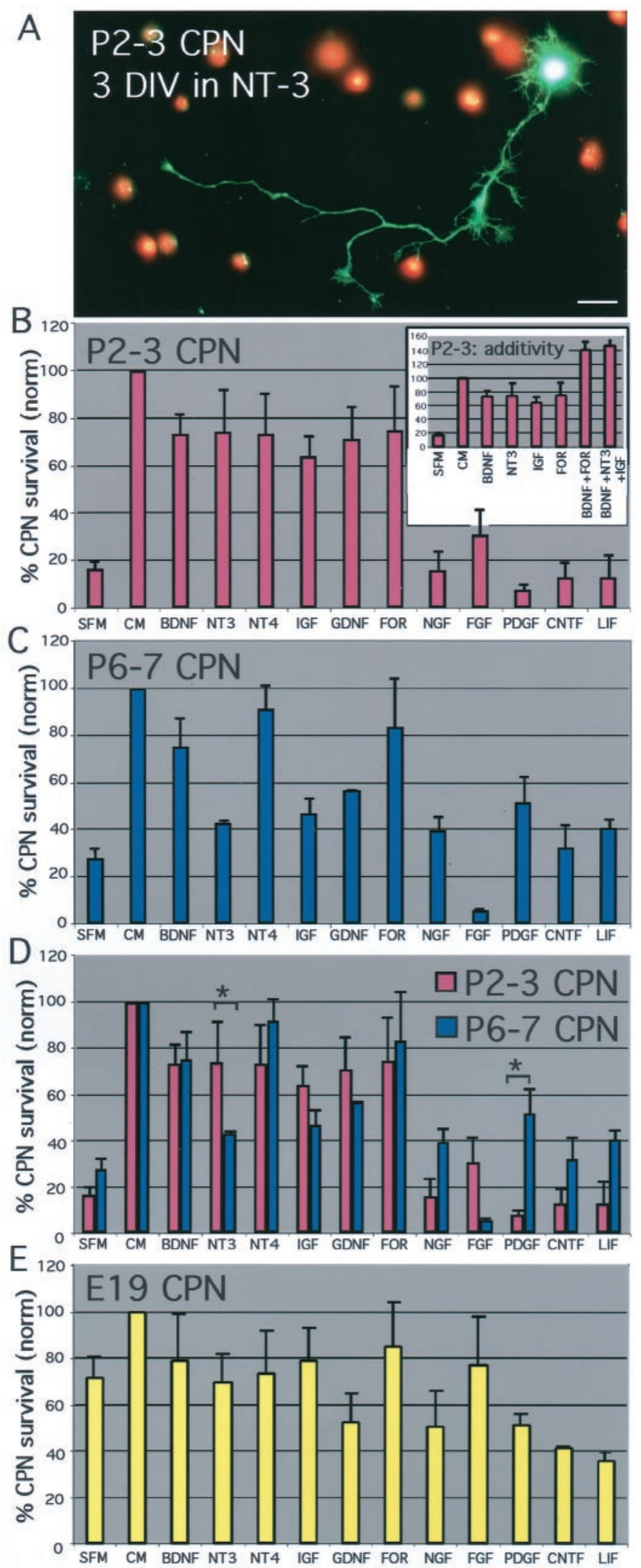

Figure 6. Specific neurotrophic factors and activity promote CPN survival in a stage-specific manner. $A, \mathrm{P} 2-\mathrm{P} 3 \mathrm{CPN}$ after 3 DIV in NT-3, displaying typical projection neuron morphology, labeled with the vital dye calcein (green). Neighboring dead cells are visualized with ethidium homodimer (red). B, Specific peptide factors promoted P2-P3 CPN sur-
RGCs, depolarization or pharmacological elevation of cAMP is required for the survival effect of neurotrophic factors (MeyerFranke et al., 1995). Cerebellar granule cells have been isolated to $90-95 \%$ purity by size fractionation and selective culture conditions (Hatten, 1985); they have also been shown to display stage-specific requirements for peptide growth factors (Segal et al., 1992; Lin and Bulleit, 1997) and a dependence on activity in culture (Gallo et al., 1987).

As a result of their experiments with RGCs, Meyer-Franke et al. (1995) proposed that the survival requirements of CNS neurons are fundamentally different from those of PNS neurons in that the CNS neuron class of RGCs requires both peptide factors and activity, in the form of depolarization or cAMP elevation, whereas peripheral neurons require only single peptide factors for survival. They speculated that the more complex survival requirements of CNS neurons may reflect their need to be integrated into neuronal circuits in the brain and their dependence on factors derived from their multiple targets. McAllister et al. (1996) have similarly reported that neocortical neurons require activity to respond to neurotrophic factors. One mechanism for this synergistic action of activity and growth factors, e.g., BDNF, is the cAMP-mediated recruitment of TrkB receptors to the neuronal cell membrane (Meyer-Franke et al., 1998). Another, complementary mechanism was proposed by Ghosh et al. (1994), who demonstrated that BDNF is required for the survival effect of depolarization-induced activation of voltage-sensitive calcium channels in E17-E18 cortical neurons in culture.

In contrast to the findings of Meyer-Franke et al. (1995), our studies demonstrate survival effects of single neurotrophic factors, and of forskolin, alone. This discrepancy may be attributable to the difference in serum-free conditions; our serum-free medium, containing the medium supplement B27, had a small but reproducible survival effect in the absence of exogenous peptide factors. Survival of CPN in B27-containing serum-free medium is significantly increased compared with that in serum-free medium containing a different medium supplement, N2 (17\% survival of $\mathrm{P} 2-\mathrm{P} 3 \mathrm{CPN}$ in B27-containing SFM vs $0 \%$ in N2-containing medium after 2 DIV). The survival-promoting activity of B27 in our serum-free medium is well below saturation, and the addition of peptide factors or $5 \mu \mathrm{M}$ forskolin to the medium has a robust additional survival effect. However, in accordance with the hypothesis put forward by Meyer-Franke et al. (1995), the activity of B27 in our experiments may potentiate the effects of neurotrophic factors and of forskolin on CPN in serum-free conditions.

Although our data clearly demonstrate the ability of specific growth factors to promote the survival of postnatal CPN in culture after axotomy, it remains to be directly determined whether these factors play a physiologic role in the control of CPN survival in vivo. Expression studies indicate that these

vival in serum-free conditions. Neuronal survival values are normalized to survival in cortical cell-conditioned medium (defined as 100\%). Error bars are SEM. FOR, Forskolin. Inset, The survival effects of the peptide factors, and of forskolin, on P2-P3 CPN survival are additive. $C$, Specific peptide factors promoted $\mathrm{P} 6-\mathrm{P} 7 \mathrm{CPN}$ survival in serum-free conditions. Survival values are normalized as in $B$. $D$, Comparative summary of the survival data from $B$ and $C$, highlighting the stage-specific differences in peptide trophic support. Asterisks denote differences in survival effects that are statistically significant $(p<0.05)$ between P2-P3 CPN and P6-P7 CPN. E, Specific peptide factors did not improve E19 CPN survival compared with survival in SFM alone. Survival values are normalized as in $B$. Scale bar, $20 \mu \mathrm{m}$. 
growth factors are expressed appropriately for such a role in vivo: BDNF (Fukumitsu et al., 1997; Yan et al., 1997), NT-3 (Fukumitsu et al., 1997), NT-4/5 (Timmusk et al., 1993), IGF-1 (Bondy, 1991), and GDNF (Choi-Lundberg and Bohn, 1995; Pochon et al., 1997) are all expressed in embryonic and/or early postnatal cortex. Our data show that CPN express at least TrkB and IGF-1 receptors in vivo, as well as in vitro. Previous studies have established roles for several of these trophic factors in the control of heterogeneous neocortical neuronal survival during development in vivo, raising the possibility that these factors act on $\mathrm{CPN}$ specifically. TrkB-/- mice exhibit increased numbers of pyknotic nuclei in cortical cells in layers II/III, V, and VI (Alcantara et al., 1997). Xu et al. (2000) selectively removed TrkB from cortical pyramidal neurons and demonstrated that these neurons undergo increased cell death postnatally and display abnormal dendritic arborization. Dissociated neocortical neurons from TrkB-/mice survive less well and differentiate more slowly and less fully than wild-type neocortical neurons in culture and after transplantation (Gates et al., 2000). Neurons from these TrkB-/- mice, transplanted into $\mathrm{P} 0$ wild-type mice, undergo delayed integration and adopt abnormal morphologies (Gates et al., 2000), suggesting a role for TrkB ligands in the control of neocortical neuron differentiation, as well as survival. IGF-1, when overexpressed in transgenic mice, leads to an increase in cortical volume, neuronal size, and neuronal number in certain regions (Gutierrez-Ospina et al., 1996). The current in vitro results are also consistent with the previous finding that, in adult cortex undergoing targeted CPN neurodegeneration, local interneurons upregulate BDNF, NT-3, and NT-4/5 (but not NGF, bFGF, CNTF, or LIF) mRNA (Wang et al., 1998). It is under these conditions of targeted CPN neurodegeneration and neurotrophin upregulation, but not control conditions, that transplanted precursor cells differentiate into CPN. These data in vivo support the hypothesis that BDNF, NT-3, and NT-4/5 are specifically required for the differentiation and survival of CPN in vivo, as well as in vitro.

These studies demonstrate for the first time the stage-specific control by peptide growth factors over the survival of a specific cortical neuronal lineage. Understanding the molecular factors that control the survival of a distinct projection neuron lineage is important for the basic understanding of neocortical development, plasticity, organization, and function. The elucidation of these controls may also contribute to a better understanding of the vulnerabilities of long-distance projection neurons, which are susceptible to dysgenesis and degeneration in a variety of neurodevelopmental and neurodegenerative disorders, and may provide an important step toward repair or regeneration of specific neuronal circuits in the neocortex via the directed differentiation of immature neural precursors.

\section{REFERENCES}

Alcantara S, Frisen J, del Rio JA, Soriano E, Barbacid M, Silos-Santiago I (1997) TrkB signaling is required for postnatal survival of CNS neurons and protects hippocampal and motor neurons from axotomyinduced cell death. J Neurosci 17:3623-3633.

Barres BA, Silverstein BE, Corey DP, Chun LLY (1988) Immunological, morphological, and electrophysiological variation among retinal ganglion cells purified by panning. Neuron 1:791-803.

Bondy CA (1991) Transient IGF-1 gene expression during the maturation of functionally related central projection neurons. J Neurosci 11:3442-3455.

Choi-Lundberg DL, Bohn MC (1995) Ontogeny and distribution of glial cell-derived neurotrophic factor (GDNF) mRNA in rat. Brain Res Dev Brain Res 85:80-88.

Conti F, Manzoni T (1994) The neurotransmitters and postsynaptic actions of callosally projecting neurons. Behav Brain Res 64:37-53.
Egaas B, Courchesne E, Saitoh O (1995) Reduced size of corpus callosum in autism. Arch Neurol 52:794-801.

Floeter MK, Jones EG (1985) The morphology and phased outgrowth of callosal axons in the fetal rat. Brain Res 354:7-18.

Fukumitsu H, Furukawa Y, Tsusaka M, Kinukawa H, Nitta A, Nomoto H, Mima T, Furukawa S (1997) Simultaneous expression of brain-derived neurotrophic factor and neurotrophin-3 in Cajal-Retzius, subplate and ventricular progenitor cells during early development stages of the rat cerebral cortex. Neuroscience 84:115-127.

Gallo V, Kingsbury A, Balazs R, Jorgenson OS (1987) The role of depolarization in the survival and differentiation of cerebellar granule cells in culture. J Neurosci 7:2203-2213.

Gates MA, Tai CC, Macklis JD (2000) Neocortical neurons lacking the protein-tyrosine kinase B (TrkB) receptor display abnormal differentiation and process elongation in vitro and in vivo. Neuroscience 98:437-447.

Ghosh A, Carnahan J, Greenberg ME (1994) Requirement for BDNF in activity-dependent survival of cortical neurons. Science 263:1618-1623.

Gutierrez-Ospina G, Calikoglu AS, Ye P, D’Ercole AJ (1996) In vivo effects of insulin-like growth factor-I on the development of sensory pathways: analysis of the primary somatic sensory cortex (S1) of transgenic mice. Endocrinology 137:5485-5492.

Hampel H, Teipel SJ, Alexander GE, Horwitz B, Teichberg D, Schapiro MB, Rappaport SI (1998) Corpus callosum atrophy is a possible indicator of region-and cell type-specific neuronal degeneration in Alzheimer disease: a magnetic resonance imaging analysis. Arch Neurol 55:192-198.

Hatten ME (1985) Neuronal regulation of astroglial morphology and proliferation in vitro. J Cell Biol 100:384-396.

Hernit-Grant CS, Macklis JD (1996) Embryonic neurons transplanted to regions of targeted photolytic cell death in adult mouse somatosensory cortex re-form specific callosal projections. Exp Neurol 139:131-142.

Ivy GO, Killackey HP (1982) Ontogenetic changes in the projections of neocortical neurons. J Neurosci 6:735-743.

Jackson CE, Bryan WW (1998) Amyotrophic lateral sclerosis. Semin Neurol 18:27-39.

Leavitt BR, Hernit-Grant CS, Macklis JD (1999) Mature astrocytes transform into transitional radial glia within adult mouse neocortex that supports directed migration of transplanted immature neurons. Exp Neurol 157:43-57.

Lin X, Bulleit RF (1997) Insulin-like growth factor I (IGF-I) is a critical trophic factor for developing cerebellar granule cells. Dev Brain Res 99:232-242.

Macklis JD (1993) Transplanted neocortical neurons migrate selectively into regions of neuronal degeneration produced by chromophoretargeted laser photolysis. J Neurosci 13:3848-3863.

Macklis JD, Quattrochi JJ (1991) Restricted diffusion and stability of carbachol-fluorescent nanospheres in-vivo. NeuroReport 2:247-250.

Magavi SS, Leavitt BR, Macklis JD (2000) Induction of neurogenesis in the neocortex of adult mice. Nature 405:951-955.

McAllister AK, Katz LC, Lo DC (1996) Neurotrophin regulation of cortical dendritic growth requires activity. Neuron 17:1057-1064

Meyer-Franke A, Kaplan MR, Pfrieger FW, Barres BA (1995) Characterization of the signaling interactions that promote the survival and growth of developing retinal ganglion cells in culture. Neuron 15:805-819.

Meyer-Franke A, Wilkinson GA, Kruttgen A, Hu M, Munro E, Hanson MG, Reichardt LF, Barres BA (1998) Depolarization and cAMP elevation rapidly recruit TrkB to the plasma membrane of CNS neurons. Neuron 21:681-693.

Pearson RCA, Esiri MM, Hiorns RW, Wilcock GK, Powell TP (1985) Anatomical correlates of the distribution of the pathological changes in the neocortex in Alzheimer's disease. Proc Natl Acad Sci USA 82:4531-4534.

Piven J, Bailey J, Ranson BJ, Arndt S (1997) An MRI study of the corpus callosum in autism. Am J Psychiatry 154:1051-1056.

Pochon M, Menoud A, Tseng JL, Zurn AD, Aebischer P (1997) Neuronal GDNF expression in the adult rat nervous system identified by in situ hybridization. Eur J Neurosci 9:463-471.

Sapp E, Penney J, Young A, Aronin N, Vonsattel JP, DiFiglia M (1999) Axonal transport of N-terminal huntingtin suggests early pathology of corticostriatal projections in Huntington disease. J Neuropath Exp Neurol 58:165-173.

Scharff C, Kirn JR, Grossman M, Macklis JD, Nottebohm F (2000) Targeted neuronal death affects neuronal replacement and vocal behavior in adult songbirds. Neuron 25:481-492.

Segal RA, Takahashi H, McKay RD (1992) Changes in neurotrophin responsiveness during the development of cerebellar granule neurons. Neuron 9:1041-1052.

Sheen VL, Macklis JD (1995) Targeted neocortical cell death in adult mice guides migration and differentiation of transplanted embryonic neurons. J Neurosci 15:8378-8392. 
Sheen VL, Arnold MW, Wang Y, Macklis JD (1999) Neural precursor differentiation following transplantation into neocortex is dependent on intrinsic developmental state and receptor competence. Exp Neurol 158:47-62.

Shin JJ, Fricker-Gates RA, Perez FA, Leavitt BR, Zurakowski D, Macklis JD (2000) Transplanted neuroblasts differentiate appropriately into projection neurons with correct neurotransmitter and receptor phenotype in neocortex undergoing targeted projection neuron degeneration. J Neurosci 20:7404-7416.

Snyder EY, Yoon C, Flax JD, Macklis JD (1997) Multipotent neural precursors can differentiate toward replacement of neurons undergoing targeted apoptotic degeneration in adult mouse neocortex. Proc Natl Acad Sci USA 94:11663-11668.

Spreafico R, Frassoni C, Arcelli P, Selvaggio M, de Biassi S (1995) In situ labeling of apoptotic cell death in the cerebral cortex and thalamus of rats during development. J Comp Neurol 363:281-295.

Timmusk T, Belluardo N, Metsis M, Persson H (1993) Widespread and developmentally regulated expression of neurotrophin- 4 mRNA in rat brain and peripheral tissues. Eur J Neurosci 5:605-613.

Wang Y, Sheen VL, Macklis JD (1998) Cortical interneurons upregulate neurotrophins in vivo in response to targeted apoptotic degeneration of neighboring pyramidal neurons. Exp Neurol 154:389-402.

Xu B, Zang K, Ruff NL, Zhang YA, McConnell SK, Stryker MP, Reichardt LF (2000) Cortical degeneration in the absence of neurotrophin signaling: dendritic retraction and neuronal loss after removal of the receptor TrkB. Neuron 26:233-245.

Yamauchi H, Fukuyama H, Nagahama Y, Katsumi Y, Dong Y, Hayashi T, Konishi J, Kimura J (1988) Atrophy of the corpus callosum, cortical hypometabolism, and cognitive impairment in corticobasal degeneration. Arch Neurol 55:609-614.

Yan Q, Rosenfeld RD, Matheson CR, Hawkins N, Lopez OT, Bennett L, Welcher AA (1997) Expression of brain-derived neurotrophic factor protein in the adult rat nervous system. Neuroscience 78:431-448. 